\title{
Space-Time Block Coding (STBC) for Multiple Transmit Antennas over Time Selective Fading Channels
}

\author{
Ramaswamy T. \\ Assistant Professor \\ MREC, Hyderabad
}

\author{
K. Chennakesava Reddy PhD \\ Principal \\ BITSW, Hyderabad
}

\begin{abstract}
A study of space-time block coding in analytical formmultiple input and multiple output (STBC-MIMO) system was done in various cases. Initially, a basic Alamouti scheme for encoding was illustrated. Then, the performance of proposed STBC-MIMO was studied under varying a number of transmitting antennas, varying number of receiving antennas, varying channels, various modulations and also at varying rates. The simulation results were obtained for all cases. At each and every stage, a new encoding matrix was derived.
\end{abstract}

\section{Keywords}

Space-Time Block Coding, OSTBC, Rayleigh fading channel, AWGN, MIMO, BER.

\section{INTRODUCTION}

In digital wireless communication required for reliability, quality and speed has tempted the notice of huge communication networks and technologist community, to contain a look at the MIMO (multiple input multiple output) technology, plays the main role to fulfill their requirements [1]. Now a day's wireless networks are used generally: wireless mesh networks (WMNs), personal area networks (PANs), cellular networks, wireless Local Area Networks (WLANs) and other type of networks like wireless sensor networks (WSNs), and now a day's these networks demand growing to have twisted spectrum into a valuable resource. Because of this cause, these approaches require anytime to set additional bits per Hz. The main solution the researcher's has fixed a notice to utilize multiple antennas at receiver (RX) and as well as a transmitter (TX). To get back, winters in [2] the MIMO used for raising capacity dates. This type of approach is called MIMO (Multiple-Input Multiple-Output) method. Here it is very important to note is that every antenna element on an MIMO method manages in the similar frequency, and it doesn't need additional bandwidth. Also for fair evolution, the single antenna system is greater than or equal to the total power via all antenna elements, i.e.

$$
\sum_{k=1}^{N} p_{k} \leq P
$$

If system was having a single antenna at that time $\mathrm{P}$ is power, $\mathrm{N}$ is total antenna elements number, and pk is allocated power via kth antenna element [4]. Efficiently, (1) MIMO doesn't require more power because it has multiple antenna elements. A number of advantages are there in MIMO model, but the only disadvantage is, when MIMO rank increases, the design of model becomes more complex. It can be realized by dividing a single into multiple models, called diversity coding. Diversity coding techniques used high range communication of MIMO model brings extra complexity. This paper gives a complete explanation on MIMO techniques involves the system model, channel models, and capacity analysis. After that importance is given to special diversity, particularly to STBC (space-time block codes). In this STBC, the concentration on orthogonal STBC and Alamouti STBC for three as well as four transmits antennas and at last shown analysis and simulation results. The remaining of paper is as follows: MIMO system model complete details shown in Section 2, STBC transmitting antennas models for two, three, four and eight are shown in Section 3, simulation results give Section 4 and finally, Section 5 gives the conclusions.

\section{MIMO SYSTEM MODEL}

Conventional wireless techniques are precious by multipath broadcast. In MIMO, but, this multipath result is demoralized to advantage the user. In fact, an occurrence of the rich multipath divide of parallel stream depended on. The cause for this result will be noticeable in system model explain in Section II-A.

\subsection{System Model}

MIMO model was having three elements those are the transmitter (TX), channel $(\mathrm{H})$, receiver $(\mathrm{RX})$. In this, Nt represents the transmitter of a number of antennas, and $\mathrm{Nr}$ represents the receivers of a number of antennas. Fig.1 illustrates the MIMO system model block diagram. It is significant that system model were explained in the channel model. For e.g. the multiple inputs are placed at the input of the channel $(\mathrm{H})$, and multiple output were placed at the output of the channel $(\mathrm{H})$.

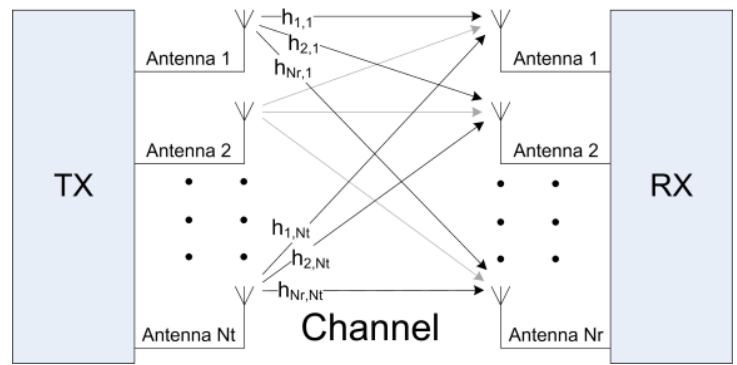

Fig. 1. MIMO (Multiple-Input Multiple-Output) system block diagram.

$\mathrm{N}_{\mathrm{t}}$ is channel inputs, and $\mathrm{N}_{\mathrm{r}}$ is channel outputs is

The channel with $\mathrm{N}_{\mathrm{r}}$ outputs and $\mathrm{N}_{\mathrm{t}}$ inputs is denoted as a $\mathrm{N}_{\mathrm{r}} \mathrm{X}$ $\mathrm{N}_{\mathrm{t}}$ matrix:

$$
H=\left(\begin{array}{ccc}
h_{1,1} h_{1,2} & \cdots & h_{1}, N_{t} \\
\vdots & \ddots & \vdots \\
h_{N_{r}, 1} h_{N_{r}, 2} & \cdots & h_{N_{r}, N_{t}}
\end{array}\right)
$$


The place each and every entry $h_{i, j}$ denotes the attenuation and segment shift (transfer operate) between the jth transmitter and the ith receiver. In this paper, the MIMO channel behaves in a "quasi-static" nature, i.e. the channel changes randomly between burst to burst, however, constant inside a transmission. This is a rule which is reasonable and assumes, as it represents an indoor channel the place the time of exchange is constant and negligible in comparison with the time of a burst of data [10]. The MIMO signal can be described as

$$
\vec{r}=H \vec{s}+\vec{n}
$$

Where $\vec{r}$ the bought vector of size $\mathrm{N}_{\mathrm{r}} \mathrm{X} 1, \mathrm{H}$ is is the channel matrix of measurement $\mathrm{N}_{\mathrm{r}} \mathrm{XN}_{\mathrm{t}}, \vec{S}$ is the transmitted vector of size $N_{t} X_{1}$, and is the noise vector of size $N_{r} \times 1$. Each noise detail is on the whole modeled as independent identically disbursed (i.e. I.D.) white Gaussian noise [1], [4] with variance $\mathrm{N}_{\mathrm{t}}=(2 . \mathrm{SNR})$ [11]. A cause of this signal is as follows. The transmitted alerts are blended in the channel because they use the identical service frequency. On the receiver part, the got sign is composed of a linear mixture of every transmitted signal plus noise. The receiver can clear up for the transmitted indicators by using treating (3) as a procedure of linear equations [3]. If the channel $\mathrm{H}$ is correlated, the process of linear equations could have more unknowns than equations. One purpose correlation between indicators can occur is because of the spacing between antennas. To hinder correlation as a result of the spacing, which is spaced with a minimum value of $\lambda_{c} / 2$, where $\lambda_{c}$ is the wavelength of the service frequency [1]. The $2 \mathrm{~d}$ rationale correlation can occur is because of lack of multipath components. It's for this reason that wealthy multipath is desirable in MIMO systems. The multipath output can be understood by means of receive antenna being with an extra channel. Accordingly, the rank of an MIMO channel is defined as the number of impartial equations provided. It is most important to notice that

$$
\operatorname{Rank}(\mathrm{H}) \leq \min \left(\mathrm{N}_{\mathrm{r}}, \mathrm{N}_{\mathrm{t}}\right)
$$

And for that reason the maximum number of streams that a MIMO system can help is higher-bounded by $\min \left(\mathrm{N}_{\mathrm{r}}, \mathrm{N}_{\mathrm{t}}\right)$. When the efficiency of MIMO systems is dependent enormously on the channel matrix, it is a main signal to the channel matrix realistically. The following section supplies an overview of usual channel units used for computer simulations.

\subsection{Channel Models}

Channel units for MIMO methods will also be either simple or very elaborate, relying on the environment modeled and the desired accuracy. There are two unique tactics for modeling MIMO channels. One approach is to calculate the MIMO channel matrix consistent with a physical representation of the environment. The channel matrix in physical model would rely upon important parameters like the angle of arrival (AOA), angle of departure (AOD), and time of arrival (TOA) [12]. In [13], a physical MIMO model and provides normal physical parameters for each macro and other micro environments. As expected, these styles of deterministic models are highly complex. An additional system to MIMO model has no. of channels logically. Such a system considers all channels b/w every transmit antenna to every receiving antenna when single input single output channels. Like this system considers that channels should be i.e. I.D (independent and identically dispersed). But, it depends on environment system, this consideration is true not all the times. The reason is that MIMO channels can expertise spatial correlation between links [12]. It is viable to generate an MIMO channel with a specified correlation matrix. The channel correlation matrix is in most cases measured within the discipline, and it's tied to the environment setup equivalent to spacing between antennas, antenna element patterns, and surrounding reflectors [12].

As one of the main themes of this paper is that differentiate the presentation of dissimilar STBC methods, such that the selected channel model link doesn't involve with the presentation. Another, channel models are the case of LOS (line-of-sight) and the case of NLOS (non-line-of-sight) correspondingly.

i) Non-line-of-sight Environment: A typical model used in research to model NLOS scenarios is the Rayleigh model. The Rayleigh system considers non-line-of-sight, and with the huge amount of scatters is used for environments. The Rayleigh model has independent identically distributed (i.i.d.) complex, zero mean, unit variance channel elements and is given by [10]:

$$
h_{i, j}=\frac{1}{\sqrt{2}}(\operatorname{Normal}(0,1)+\sqrt{-1} \cdot \operatorname{Normal}(0,1)(5)
$$

This model results in an approximation that improves as the spacing between antennas become large compared to the wavelength $\lambda$.

ii) Line-of-sight Model: The MIMO channel matrix for the Line-of-sight setting is given by [12]:

$H=\sqrt{\frac{K}{1+K}} H_{L O S}+\sqrt{\frac{1}{1+K}} H_{N L O S}$

Where:

$K(d B)=10 \log _{10} \frac{P_{L O S}}{P_{N L O S}}$

In (6), HLOS is a rank-one matrix akin to the LOS element, and the HNLOS corresponds to the NLOS components. In (7), PLOS is the vigour because of the LOS element, and PNLOS is due to the power of the NLOS element [12]. The HNLOS aspect is in most cases modeled as (5) [3]. In SISO techniques, the better the ok element, the smaller the fade fringe needed. For MIMO model, the $\mathrm{K}$ facto is high, will be the more leading the rank-one HLOS element, and thus, will be the less leading the HNLOS element. But, for the setting of rich multipath, measurements and simulations showed that the Line-of-sight element infrequently controls [3].

\section{STBC PROPOSED APPROACH}

One of the methodologies for exploiting the ability in MIMO method consists of using the further variety of MIMO programs, specifically spatial range, to combat channel fading. This will be attained with a sending quantity of units of the similar information via every antenna. Even as doing these matters dropping probability understanding reduces exponentially [3]. The antennas in an MIMO approach are used for aiding a transmission of an SISO approach because the distinctive expense of is that of an SISO approach. The variety order or range obtains of an MIMO system is defined as the number of impartial receptions of the same sign. A MIMO process with $\mathrm{N}_{\mathrm{t}}$ transmit antennas and $\mathrm{N}_{\mathrm{r}}$ acquire antennas has possibly full variety (i.e. Maximum diversity) attain equal to $\mathrm{N}_{\mathrm{t}} \mathrm{N}_{\mathrm{r}}$. The dissimilar replicas transmit for developing diversity is producing with a space-time encoder it encodes a single via time with transmitting every symbol at dissimilar times. This type of coding called STC (space-time 
coding). Their simple decoding, most leading type of spacetime coding is STBC (space-time block coding). In next, a space-time block coding system is dissimilar and has contrast for presentation in Section 4.

\subsection{Alamouti's STBC}

In [14], Alamouti published his manner on transmit diversity. Historically, Alamouti's scheme was once the primary STBC [4]. The simplicity and basic structure of the Alamouti STBC have placed the scheme in each the W-CDMA and CDMA2000 standards [3]. The Alamouti STBC scheme makes use of two transmit antennas and $\mathrm{Nr}$ obtain antennas and can accomplish a maximum range order of $2 \mathrm{Nr}$ [14]. In addition to the Alamouti system has rated 1 (i.e. full rate) as each 2time intervals it transmits two symbols. After, that Alamouti system is obtained for one and two received antennas, followed with a normal term used for the decoding method for the case of receive antennas $\mathrm{N}_{\mathrm{r}}$.

\section{(i) Explanation:}

As acknowledged prior, Alamouti STBC uses two transmit antennas whatever the number of acquiring antennas. The Alamouti scheme encoding operation is obtained through (eight). In this paper, the rows of each and every coding scheme represent an extra time instant, even as the columns characterize the transmitted symbol by way of each special antenna. On this case, the first and 2nd rows signify the transmission at the first and second time instant respectively. At a time t, the logo s1 and image s2 are transmitted from antenna 1 and antenna two respectively. Assuming that each and every symbol has a period $\mathrm{T}$, and then at time $\mathrm{t}+\mathrm{T}$, the symbols are $-s_{2}^{*}$ and $s_{1}^{*}$, where $(.)^{*}$ denotes the problematic conjugate, are transmitted from antenna 1 and antenna two respectively.

$\mathcal{G}_{2}=\operatorname{time}\left(\begin{array}{cc}s_{1} & S_{2} \\ -S_{2}^{*} & -s_{1}^{*}\end{array}\right)$

\section{ii) Case of 1 Receive Antenna:}

The amount of available receiving antennas of the decoding and response of the signal are depended on. For case of one received antenna signals are [14]:

$$
\begin{aligned}
r_{1}^{(1)}= & r_{1}(t)=h_{1,1} s_{1}+h_{1,2} s_{2}+n_{1}^{(1)} \\
& r_{1}^{(2)}=r_{1}(t+T)=-h_{1,1} s_{2}^{*}+h_{1,2} s_{1}^{*}+n_{1}^{(2)}
\end{aligned}
$$

The place at one antenna acquired sign is $r_{1}$, channel switch perform is $h_{i, j}$ is transmitted antenna $j_{\text {th }}$ and acquire antenna $i_{\text {th }}$ mentioned in chapter 3 , at antennal tricky random variable $\mathrm{n}_{1}$, and represents $\mathrm{x}$ at instantaneous time $\mathrm{k}$. Got signal are transmitted before handiest to the decoder, they blended as follows [14]:

$$
\begin{aligned}
& \tilde{s}_{1}=h_{1,1}^{*} r_{1}^{(1)}+h_{1,2} r_{1}^{*(2)} \\
& \tilde{s}_{2}=h_{1,2}^{*} r_{1}^{(1)}+h_{1,1} r_{1}^{*(2)}
\end{aligned}
$$

And substituting (9) in (10) yields:

$$
\begin{aligned}
& \tilde{s}_{1}=\left(\alpha_{1,1}^{2}+\alpha_{1,2}^{2}\right) s_{1}+h_{1,1}^{*} n_{1}^{(1)}+h_{1,2} n_{1}^{*(2)} \\
& \tilde{s}_{2}=\left(\alpha_{1,1}^{2}+\alpha_{1,2}^{2}\right) s_{2}-h_{1,1} n_{1}^{*(2)}+h_{1,2}^{*} n_{1}^{(1)}
\end{aligned}
$$

Where $\alpha_{i, j}^{2}$ is the squared magnitude of the channel transfer function $\mathrm{h}_{\mathrm{i}, \mathrm{j}}$. The calculated $\tilde{s}_{1}$ and $\tilde{s}_{2}$ are then transmit to an ML (Maximum Likelihood) decoder to approximation the sender symbols $\mathrm{s}_{1}$ and $\mathrm{s}_{2}$ respectively [14].

\section{iii). Case of two Receive Antennas:}

For the case considered with two receive antennas, the received symbols are represented as [14]:

$$
\begin{gathered}
r_{1}^{(1)}=h_{1,1} s_{1}+h_{1,2} s_{2}+n_{1}^{(1)} \\
r_{1}^{(2)}=-h_{1,1} s_{2}^{*}+h_{1,2} s_{1}^{*}+n_{1}^{(2)} \\
r_{2}^{(1)}=h_{2,1} s_{1}+h_{2,2} s_{2}+n_{2}^{(1)} \\
r_{2}^{(2)}=-h_{2,1} s_{2}^{*}+h_{2,2} s_{1}^{*}+n_{2}^{(2)}
\end{gathered}
$$

and the combined signals are [14]:

$$
\begin{gathered}
\tilde{s}_{1}=h_{1,1}^{*} r_{1}^{(1)}+h_{1,2} r_{1}^{*(2)}+h_{2,1}^{*} r_{2}^{(1)}+h_{2,2} r_{2}^{*(2)} \\
\tilde{s}_{2}=h_{1,2}^{*} r_{1}^{(1)}+h_{1,1} r_{1}^{*(2)}+h_{2,2}^{*} r_{2}^{(1)}+h_{1,1} r_{2}^{*(2)}
\end{gathered}
$$

Which, after substituting (11) becomes:

$$
\begin{aligned}
& \tilde{s}_{1}=\left(\alpha_{1,1}^{2}+\alpha_{1,2}^{2}+\alpha_{2,1}^{2}+\alpha_{2,2}^{2}\right) s_{1}+h_{1,1}^{*} n_{1}^{(1)}+ \\
& h_{1,2} n_{1}^{*(2)}+h_{2,1}^{*} n_{2}^{(1)}+h_{2,2} n_{2}^{*(2)} \\
& \begin{aligned}
\tilde{s}_{2}=\left(\alpha_{1,1}^{2}+\alpha_{1,2}^{2}\right. & \left.+\alpha_{2,1}^{2}+\alpha_{2,2}^{2}\right) s_{2}-h_{1,1} n_{1}^{*(2)} \\
& +h_{1,2}^{*} n_{1}^{(1)}-h_{2,1} n_{2}^{*(2)}+h_{2,2}^{*} n_{2}^{(1)}
\end{aligned}
\end{aligned}
$$

iv). Decoding decision statistic for $\mathrm{Nr}$ receives antennas:

The ML decoder decision statistic decodes in desire of $\mathrm{s} 1$ and s2 over all possible values of s1 and s2 such that (15) and (16) are minimized where is given with the aid of (17) for $\mathrm{Nt}=2$ [15], [11].

$$
\begin{aligned}
& \left|\left[\sum_{i=1}^{N_{r}}\left(r_{i}^{(1)} h_{i, 1}^{*}+r_{i}^{*(2)} h_{i, 2}\right)\right]-s_{1}\right|^{2}+\psi\left|s_{1}\right|^{2}(15) \\
& \left|\left[\sum_{i=1}^{N_{r}}\left(r_{i}^{(1)} h_{i, 2}^{*}-r_{i}^{*(2)} h_{i, 1}\right)\right]-s_{2}\right|^{2}+\psi\left|s_{2}\right|^{2}(16) \\
& \psi=\left(-1+\sum_{i=1}^{N_{r}} \sum_{j=1}^{N_{t}}\left|h_{i, j}\right|^{2}\right)
\end{aligned}
$$

Alamouti STBC does not require CSI at the transmitter. Also, the Alamouti STBC can be utilized with two transmitted antennas and 1 obtain antenna even as engaging in the entire variety of 2. That is a foremost characteristic of Alamouti STBC as it reduces the outcomes of fading at cellular stations with best requiring additional antenna factors on the base station, the place it's less expensive than having multiple antennas on the receivers [14]. However, if having more antennas at the receivers is just not a difficulty, this scheme can be utilized with two transmitted antennas and $\mathrm{Nr}$ acquires antennas whilst undertaking a $2 \mathrm{Nr}$ full diversity. The case space time block codes for $\mathrm{N}_{\mathrm{T}}>2$ are discussed within the following part.

\subsection{Orthogonal Space-Time Block Codes}

The Alamouti scheme mentioned in chapter III. It is a part of a common classification of STBCs often called orthogonal space-Time Block Codes (OSTBCs) [4]. The authors of [15] observe the mathematical framework of orthogonal designs to construct each real and problematic orthogonal code that 
acquire full range. For the case of actual orthogonal codes, it has been shown that a full fee code will also be developed [15]. Nevertheless, for the case of complicated orthogonal codes, it's unknown if a full cost and entire variety codes exist for NT>2 [3]. Elaborate modulations methods are of interest in this paper and hence real orthogonal codes aren't discussed. In subsequent sections, the full diversity complex orthogonal codes presented in [15] for special premiums are briefly introduced.

\section{1) Orthogonal $\mathrm{STBC}$ for $\mathrm{N}_{\mathrm{t}}=3$ :}

For the case of three transmit antennas. Assemble block codes for the with half of and $3 /$ four coding expense and whole diversity $3 \mathrm{Nr}$.

i). $\mathrm{Nt}=3$ with Rate $1 / 2$ :

The full diversity, rate $1 / 2$ code for $\mathrm{Nt}=3$ are given by [15], [11]:

$\mathcal{G}_{3}=\left(\begin{array}{ccc}s_{1} & s_{2} & s_{3} \\ -s_{2} & s_{1} & -s_{4} \\ -s_{3} & s_{4} & s_{1} \\ -s_{4} & -s_{3} & s_{2} \\ s_{1}^{*} & s_{2}^{*} & s_{3}^{*} \\ -s_{2}^{*} & s_{1}^{*} & -s_{4}^{*} \\ -s_{3}^{*} & s_{4}^{*} & s_{1}^{*} \\ -s_{4}^{*} & -s_{3}^{*} & s_{2}^{*}\end{array}\right)$

This code transmits four symbols each eight time intervals, and for that reason has expense half. The choice metric to shrink via the decoder for detecting s1, s2, s3, s4 are given with the aid of (20), (21), (22), (23) respectively the place

$\xi=\left(-1+2 \sum_{i=1}^{N_{r}} \sum_{j=1}^{N_{t}}\left|h_{i, j}\right|^{2}\right)$

For $\mathrm{N}_{\mathrm{t}}=3[11]$.

$\mid\left[\sum_{i=1}^{N_{r}}\left(r_{i}^{(1)} h_{i, 1}^{*}+r_{i}^{(2)} h_{i, 2}^{*}+r_{i}^{(3)} h_{i, 3}^{*}+r_{i}^{*(5)} h_{i, 1}+\right.\right.$

$\left.\left.r_{i}^{*(6)} h_{i, 2}+r_{i}^{*(7)} h_{i, 3}\right)\right]-\left.s_{1}\right|^{2}+\xi\left|s_{1}\right|^{2}$

$\mid\left[\sum_{i=1}^{N_{r}}\left(r_{i}^{(1)} h_{i, 2}^{*}-r_{i}^{(2)} h_{i, 1}^{*}+r_{i}^{(4)} h_{i, 3}^{*}+r_{i}^{*(5)} h_{i, 2}-\right.\right.$

$\left.\left.r_{i}^{*(6)} h_{i, 1}+r_{i}^{*(8)} h_{i, 3}\right)\right]-\left.s_{1}\right|^{2}+\xi\left|s_{2}\right|^{2}$

$\mid\left[\sum_{i=1}^{N_{r}}\left(r_{i}^{(1)} h_{i, 3}^{*}-r_{i}^{(3)} h_{i, 1}^{*}+r_{i}^{(4)} h_{i, 2}^{*}+r_{i}^{*(5)} h_{i, 3}-\right.\right.$

$\left.\left.r_{i}^{*(7)} h_{i, 1}-r_{i}^{*(8)} h_{i, 2}\right)\right]-\left.s_{3}\right|^{2}+\xi\left|s_{3}\right|^{2}$

$\mid\left[\sum_{i=1}^{N_{r}}\left(-r_{i}^{(2)} h_{i, 3}^{*}+r_{i}^{(3)} h_{i, 2}^{*}-r_{i}^{(4)} h_{i, 1}^{*}-r_{i}^{*(6)} h_{i, 3}+\right.\right.$

$\left.\left.r_{i}^{*(7)} h_{i, 2}-r_{i}^{*(8)} h_{i, 1}\right)\right]-\left.s_{4}\right|^{2}+\xi\left|s_{4}\right|^{2}$

ii) $\mathrm{Nt}=3$ with Rate $3 / 4$ :

A higher rate code with $\mathrm{Nt}=3$ is given by [15], [11]

$\mathcal{H}_{3}=\left(\begin{array}{ccc}s_{1} & s_{2} & \frac{s_{3}}{\sqrt{2}} \\ -s_{2}^{*} & s_{1}^{*} & \frac{s_{3}}{\sqrt{2}} \\ \frac{s_{3}^{*}}{\sqrt{2}} & \frac{s_{3}^{*}}{\sqrt{2}} & \frac{-s_{1}-s_{1}^{*}+s_{2}-s_{2}^{*}}{2} \\ \frac{s_{3}^{*}}{\sqrt{2}} & -\frac{s_{3}^{*}}{\sqrt{2}} & \frac{s_{2}+s_{2}^{*}+s_{1}-s_{1}^{*}}{2}\end{array}\right)$

As can be determined, (23) transmits 3 symbols each 4 time intervals, and therefore has rate three/4. The choice statistic to diminish for detecting $s 1, s 2$, and $s 3$ are given by (25), (26), and

respectively: $\mid\left[\sum_{i=1}^{N_{r}}\left(r_{i}^{(1)} h_{i, 1}^{*}+r_{i}^{*(2)} h_{i, 2}+\right.\right.$

$\left.\left.\frac{\left(r_{i}^{(4)}-r_{i}^{(3)}\right) h_{i, 3}^{*}}{2}-\frac{\left(r_{i}^{(3)}+r_{i}^{(4)}\right)^{*} h_{i, 3}}{2}\right)\right]-\left.s_{1}\right|^{2}+\psi\left|s_{1}\right|^{2}$

$\mid\left[\sum_{i=1}^{N_{r}}\left(r_{i}^{(1)} h_{i, 2}^{*}-r_{i}^{*(2)} h_{i, 1}+\frac{\left(r_{i}^{(4)}+r_{i}^{(3)}\right) h_{i, 3}^{*}}{2}+\right.\right.$

$\left.\left.\frac{\left(-r_{i}^{(3)}+r_{i}^{(4)}\right)^{*} h_{i, 3}}{2}\right)\right]-\left.s_{2}\right|^{2}+\psi\left|s_{2}\right|^{2}$

$\mid\left[\sum_{i=1}^{N_{r}}\left(\frac{\left(r_{i}^{(1)}+r_{i}^{(2)}\right) h_{i, 3}^{*}}{\sqrt{2}}+\frac{r_{i}^{*(3)}\left(h_{i, 1}+h_{i, 2}\right)}{\sqrt{2}}+\frac{r_{i}^{*(4)}\left(h_{i, 1}-h_{i, 2}\right)}{\sqrt{2}}\right)\right]-$ $\left.s_{3}\right|^{2}+\psi\left|s_{3}\right|^{2}$

2) Orthogonal STBC for $\mathrm{Nt}=4$ :

For the case of 4 transmit antennas, [15] provide block codes of price $1=2$ and $3=4$, each of which have full diversity $4 \mathrm{Nr}$.

i) $\mathrm{Nt}=4$ with Rate $1=2$ : In the case of 4 transmit antennas, the rate $1 / 2$ code block is given by [15], [11]:

$\mathcal{G}_{4}=\left(\begin{array}{cccc}s_{1} & s_{2} & s_{3} & s_{4} \\ -s_{2} & s_{1} & -s_{4} & s_{3} \\ -s_{3} & s_{4} & s_{1} & -s_{2} \\ -s_{4} & -s_{3} & s_{2} & s_{1} \\ s_{1}^{*} & s_{2}^{*} & s_{3}^{*} & s_{4}^{*} \\ -s_{2}^{*} & s_{1}^{*} & -s_{4}^{*} & s_{3}^{*} \\ -s_{3}^{*} & s_{4}^{*} & s_{1}^{*} & -s_{2}^{*} \\ -s_{4}^{*} & -s_{3}^{*} & s_{2}^{*} & s_{1}^{*}\end{array}\right)$

Where, just like (18), has price $1 / 2$ as 4 symbols are transmitted in 8 time intervals. To decode, the ML decoder minimizes the selection metric (22), (23), (24), and (25) for decoding $\mathrm{s} 1, \mathrm{~s} 2, \mathrm{~s} 3$, and $\mathrm{s} 4$ respectively the place is given by means of $(18)$ for $\mathrm{Nt}=$ four [11]. The decoding selection metric (32) for decoding s4 differs from that of [11] considering that the writer found out a mistake within the metric offered.

$$
\begin{aligned}
& \mid\left[\sum _ { i = 1 } ^ { N _ { r } } \left(r_{i}^{(1)} h_{i, 1}^{*}+r_{i}^{(2)} h_{i, 2}^{*}+r_{i}^{(3)} h_{i, 3}^{*}+r_{i}^{*(4)} h_{i, 4}+\right.\right. \\
& \left.\left.r_{i}^{*(5)} h_{i, 1}+r_{i}^{*(6)} h_{i, 2}+r_{i}^{*(7)} h_{i, 3}+r_{i}^{*(8)} h_{i, 4}\right)\right]-\left.s_{1}\right|^{2}+ \\
& \xi\left|s_{1}\right|^{2}
\end{aligned}
$$

$$
\begin{aligned}
& \mid\left[\sum _ { i = 1 } ^ { N _ { r } } \left(r_{i}^{(1)} h_{i, 2}^{*}-r_{i}^{(2)} h_{i, 1}^{*}-r_{i}^{(3)} h_{i, 4}^{*}+r_{i}^{(4)} h_{i, 3}^{*}+\right.\right. \\
& \left.\left.r_{i}^{(5)} h_{i, 2}-r_{i}^{*(6)} h_{i, 1}-r_{i}^{*(7)} h_{i, 4}+r_{i}^{*(8)} h_{i, 3}\right)\right]-\left.s_{2}\right|^{2}+ \\
& \xi\left|s_{2}\right|^{2}(30) \\
& \mid\left[\sum _ { i = 1 } ^ { N _ { r } } \left(r_{i}^{(1)} h_{i, 3}^{*}+r_{i}^{(2)} h_{i, 4}^{*}-r_{i}^{(3)} h_{i, 1}^{*}-r_{i}^{*(4)} h_{i, 2}^{*}+\right.\right. \\
& \left.\left.r_{i}^{*(5)} h_{i, 3}+r_{i}^{*(6)} h_{i, 4}-r_{i}^{*(7)} h_{i, 1}-r_{i}^{*(8)} h_{i, 2}\right)\right]-\left.s_{3}\right|^{2}+ \\
& \xi\left|s_{3}\right|^{2}(31) \\
& \mid\left[\sum _ { i = 1 } ^ { N _ { r } } \left(r_{i}^{(1)} h_{i, 4}^{*}-r_{i}^{(2)} h_{i, 3}^{*}+r_{i}^{(3)} h_{i, 2}^{*}-r_{i}^{(4)} h_{i, 1}^{*}+\right.\right. \\
& \left.\left.r_{i}^{*(5)} h_{i, 4}-r_{i}^{*(6)} h_{i, 3}+r_{i}^{*(7)} h_{i, 2}-r_{i}^{*(8)} h_{i, 1}\right)\right]-\left.s_{4}\right|^{2}+ \\
& \xi\left|s_{4}\right|^{2}
\end{aligned}
$$

ii). $\mathrm{Nt}=4$ with Rate $3 / 4$ :

A four transmit antenna block code with rate $3 / 4$ is given by [15], [11]: 


$$
\mathcal{H}_{4}=\left(\begin{array}{cccc}
s_{1} & s_{2} & \frac{s_{3}}{\sqrt{2}} & \frac{s_{3}}{\sqrt{2}} \\
-s_{2}^{*} & s_{1}^{*} & \frac{s_{3}}{\sqrt{2}} & -\frac{s_{3}}{\sqrt{2}} \\
\frac{s_{3}^{*}}{\sqrt{2}} & \frac{s_{3}^{*}}{\sqrt{2}} & \frac{-s_{1}-s_{1}^{*}+s_{2}-s_{2}^{*}}{2} & \frac{-s_{2}-s_{2}^{*}+s_{1}-s_{1}^{*}}{2} \\
\frac{s_{3}^{*}}{\sqrt{2}} & -\frac{s_{3}^{*}}{\sqrt{2}} & \frac{s_{2}+s_{2}^{*}+s_{1}-s_{1}^{*}}{2} & -\frac{s_{1}+s_{1}^{*}+s_{2}-s_{2}^{*}}{2}
\end{array}\right)
$$

The decision metrics to minimize for decoding $\mathrm{s}_{1}, \mathrm{~s}_{2}$, and $\mathrm{s}_{3}$ are given by (34), (35), and (36) respectively

$$
\begin{gathered}
\mid\left[\sum _ { i = 1 } ^ { N _ { r } } \left(r_{i}^{(1)} h_{i, 1}^{*}+r_{i}^{*(2)} h_{i, 2}+\frac{\left(r_{i}^{(4)}-r_{i}^{(3)}\right)\left(h_{i, 3}^{*}-h_{i, 4}^{*}\right)}{2}-\right.\right. \\
\left.\left.\frac{\left(r_{i}^{(3)}+r_{i}^{(4)}\right)^{*}\left(h_{i, 3}+h_{i, 4}\right)}{2}\right)\right]-\left.s_{1}\right|^{2}+\psi\left|s_{1}\right|^{2} \\
\mid\left[\sum _ { i = 1 } ^ { N _ { r } } \left(r_{i}^{(1)} h_{i, 2}^{*}-r_{i}^{*(2)} h_{i, 1}+\frac{\left(r_{i}^{(4)}+r_{i}^{(3)}\right)\left(h_{i, 3}^{*}-h_{i, 4}^{*}\right)}{2}+\right.\right. \\
\mid\left[\sum _ { i = 1 } ^ { N _ { r } } \left(\frac{\left(r_{i}^{(1)}+r_{i}^{(2)}\right) h_{i, 3}^{*}}{\sqrt{2}}+\frac{\left(r_{i}^{(1)}-r_{i}^{(2)}\right) h_{i, 4}}{\sqrt{2}}+\frac{r_{i}^{*(3)}\left(h_{i, 1}+h_{i, 2}\right)}{\sqrt{2}}+\right.\right. \\
\left.\left.\frac{r_{i}^{*(4)}\left(h_{i, 1}-h_{i, 2}\right)}{\sqrt{2}}\right)\right]-\left.s_{3}\right|^{2}+\psi\left|s_{3}\right|^{2}
\end{gathered}
$$

iii) $\mathrm{Nt}=8$ with Rate $1 / 2$ :

Within the case of 8 transmit antennas, the fee half code block is given with the aid of eq.(37). Similarly the real transmission matrix is given through $\mathrm{Nt}=8$

$$
G_{8}=\left(\begin{array}{cccccccc}
s_{1} & s_{2} & s_{3} & s_{4} & s_{5} & s_{6} & s_{7} & s_{8} \\
-s_{2} & s_{1} & s_{4} & -s_{3} & s_{6} & -s_{5}-s_{8} & s_{7} \\
-s_{3} & -s_{4} & s_{1} & s_{2} & s_{7} & s_{8} & -s_{5} & -s_{6} \\
-s_{4} & s_{3} & -s_{2} & s_{1} & s_{8} & -s_{7} & s_{6} & -s_{5} \\
-s_{5}^{*}-s_{6}^{*}-s_{7}^{*}-s_{8}^{*} & s_{1}^{*} & s_{2}^{*} & s_{3}^{*} & s_{4}^{*} \\
-s_{6}^{*} & s_{5}^{*} & -s_{8}^{*} & s_{7}^{*} & -s_{2}^{*} & s_{1}^{*} & -s_{4}^{*} & s_{3}^{*} \\
-s_{7}^{*} & s_{8}^{*} & s_{5}^{*} & -s_{6}^{*}-s_{3}^{*} & s_{4}^{*} & s_{1}^{*} & -s_{2}^{*} \\
-s_{8}^{*}-s_{7}^{*} & s_{6}^{*} & s_{5}^{*} & -s_{4}^{*} & s_{3}^{*} & s_{2}^{*} & s_{1}^{*}
\end{array}\right)
$$

On the receiver finish, the obtained expressions are headquartered on Alamouti's model with the simplicity of getting only actual symbols and consequently no conjugate symbol in the equations. Accordingly the acquired expressions for any number of got antennas end up $r_{1}, N_{R}=$ $r_{N_{R}} t=h_{1, N_{R}} s_{1}+h_{2, N_{R}} s_{2}+n_{1}, N_{R}$,

$$
r_{2}, N_{R}=r_{N_{R}} t+T=-h_{2, N_{R}} s_{2}+h_{1, N_{R}} s_{1}+n_{2}, N_{R},
$$

Where $n_{1, N R}, n_{2, N R}$ are independent noise samples, $h_{i, N R}$ is the channel transfer operate from the $i_{\text {th }}$ transmit antenna and NR denotes the MT acquire antenna. Acquired alerts are then mixed for two transmit antennas as

$$
\begin{gathered}
\tilde{s}_{1}=\sum_{N_{R}=1}^{M_{R}} r_{1}, N_{R} h_{1, N_{R}}+r_{2}, N_{R} h_{2, N_{R}} \\
\tilde{s}_{2}=\sum_{N_{R}=1}^{M_{R}} r_{1}, N_{R} h_{2, N_{R}}-r_{2}, N_{R} h_{1, N_{R}}
\end{gathered}
$$

Similarly, the acquired sign for four and eight transmit antennas will also be derived. Alamouti STBC don't require CSI on the transmitter and can be utilized with two transmit antennas and 1 obtain antenna with accomplishment of full variety of two. It reduces the outcomes of fading at receiver station at the rate of some additional antenna factors at the transmitter end. If having extra antennas will not be a trouble, then this scheme is correct for getting full variety of 2NR with two transmit antennas. Now that the block codes and their decoding metrics were awarded, the simulation setup is mentioned, adopted by using results and evaluation, and subsequently the conclusion.

\section{EXPERIMENTAL RESULTS}

An experimental result shows the different constant $\mathrm{Nr}, \mathrm{Nt}$ diversity order and rate for different cases of QPSK, 16-QAM and 64-QAM.

\section{Case 1: Rayleigh channel}

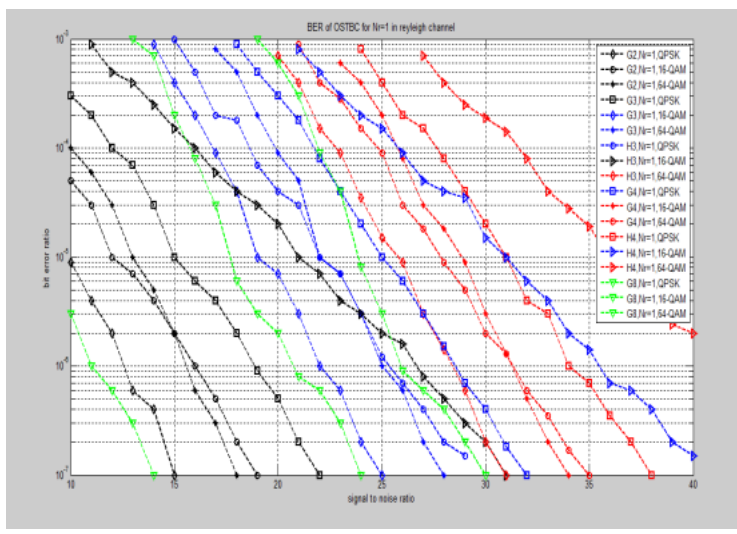

Fig.2 Bit error rate versus SNR of OSTBC for $\mathrm{Nr}=1$.

As we shown in the above figure represents the BER V/s SNR with constant $\mathrm{Nr}=1$. In this case G8 shows the best performance compared to the G2, G3, G4, H3 and H4. Compare to theG8 of QPSK G2, G3, H3, G4, H4 of QPSK is high SNR, theG8 of 16-QAM and 64-QAM G2, G3, H3, G4, H4 of 16-QAM and 64-QAM are high SNR also, so that the results shown that G8 is best performance compare to the other. G8 having the low SNR value so that it is the best performer when compare to the other.

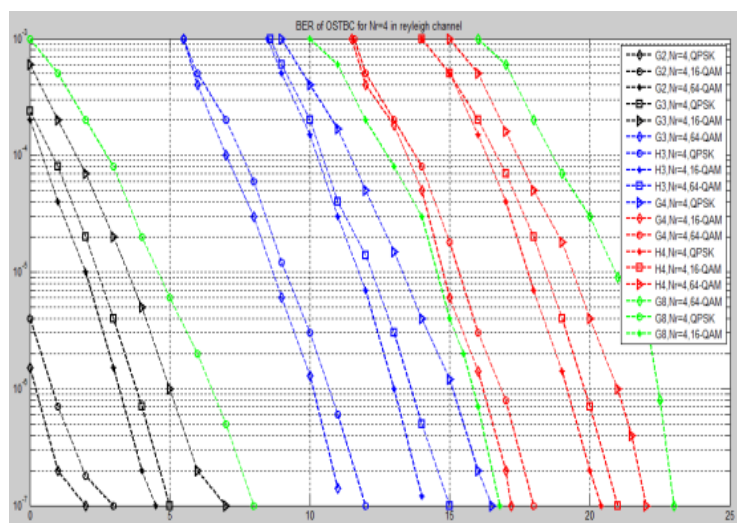

Fig.3 Bit error rate versus SNR of OSTBC for $\mathrm{Nr}=4$

As we shown in the above figure represents the BER V/s SNR with constant $\mathrm{Nr}=4$. In this case G8 shows the best performance compared to the G2,G3,G4, H3 and H4. Compare to theG8 of QPSK G2, G3, H3, G4, H4 of QPSK is low SNR, theG8 of 16-QAM and 64-QAM G2, G3, H3, G4, H4 of 16-QAM and 64-QAM are low SNR also, so that the results shown that G8 is best performance compare to the other. G8 having the high SNR value so that it is the best performer when compare to the other. 


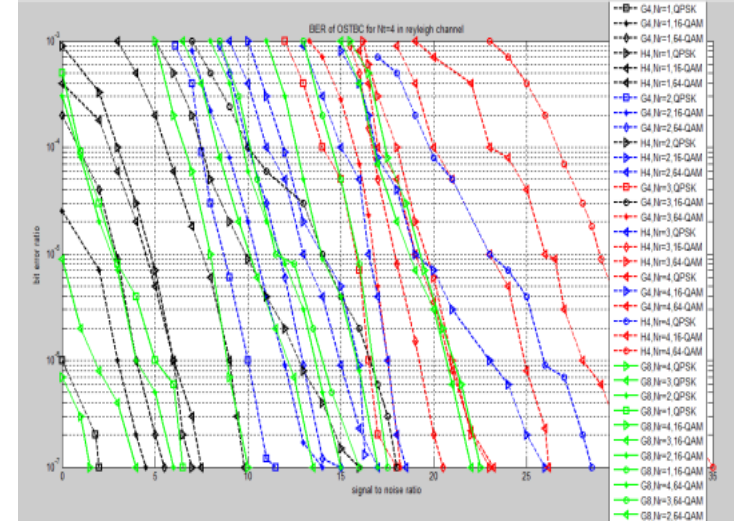

Fig.4 Bit error rate versus SNR of OSTBC for $\mathrm{Nt}=4$

As we shown in the above figure represents the BER V/s SNR with different $\mathrm{Nr}=1,2,3,4$. In this case G8 shows the best performance compared to the G2, G3, G4, H3 and H4. Compare to theG8 of QPSK G2, G3, H3, G4, H4 of QPSK is high SNR, theG8 of 16-QAM and 64-QAM G2, G3, H3, G4, H4 of 16-QAM and 64-QAM are high SNR also, so that the results shown that G8 is best performance compare to the other. G8 having the low SNR value so that it is the best performer when compare to the other.

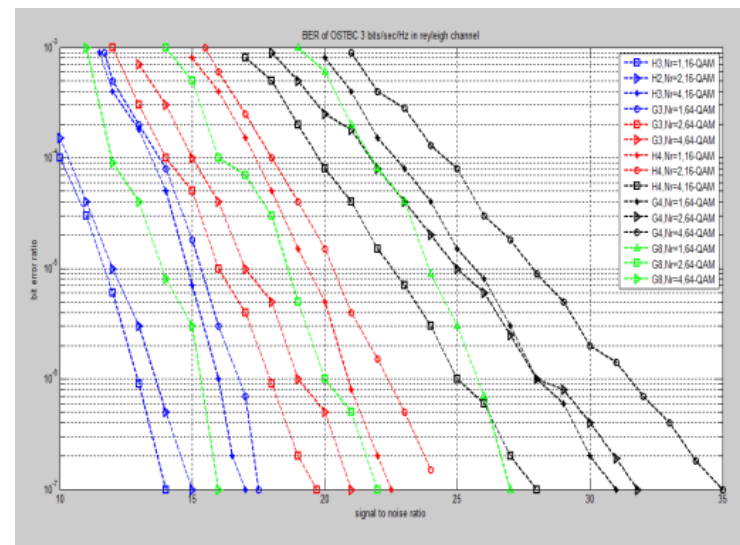

Fig.5 Bit error rate versus SNR of OSTBC at $3 \mathrm{bits} / \mathrm{sec} / \mathrm{Hz}$.

As we shown in the above figure represents the BER V/s SNR with different $\mathrm{Nr}=1,2$, 4. In this case G8 shows the best performance compared to theG3, G4. Compare to theG8, 64QAM G3, G4 of 64-QAM are low SNR, so that the results shown that G8 is best performance compare to the other. G8 having the high SNR value so that it is the best performer when compare to the other.

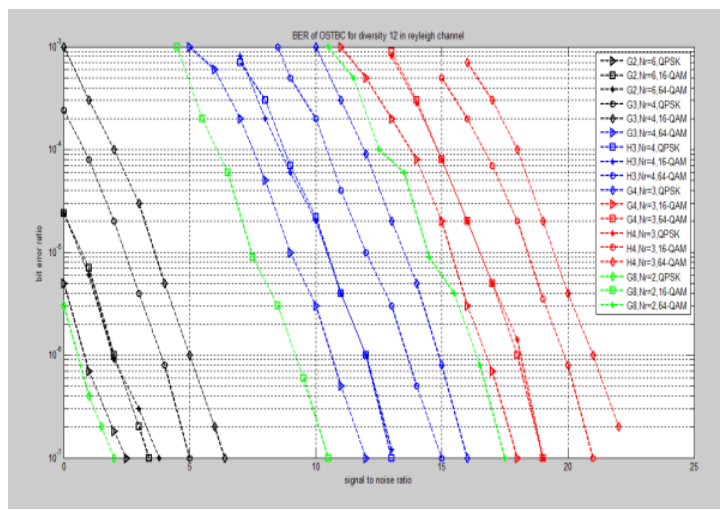

Fig.6 Bit error rate versus SNR of OSTBC with spatial diversity 12 .
As we shown in the above figure represents the BER V/s SNR with different $\mathrm{Nr}=6,4,3,2$. In this case G8 shows the best performance compared to the G2, G3, G4, H3 and H4 Compare to theG8 of QPSK G2, G3, H3, G4, H4 of QPSK is high SNR, theG8 of 16-QAM and 64-QAM G2, G3, H3, G4, H4 of 16-QAM and 64-QAM are high SNR also, so that the results shown that G8 is best performance compare to the other. G8 having the low SNR value so that it is the best performer when compare to the other. Increase the number of $\mathrm{Nt}$ transmit antennas at the base station than increasing the number of $\mathrm{Nr}$ receive antennas at all mobile stations.

\section{Case 2: Rician channel}

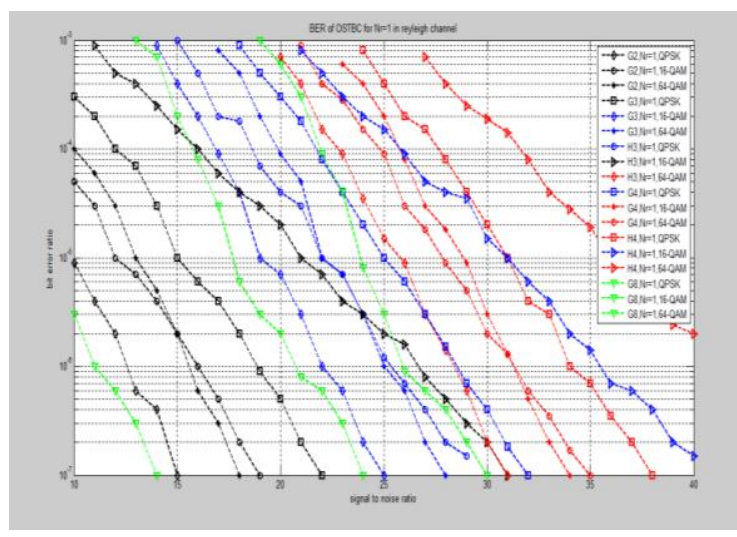

Fig.7 Bit error rate versus SNR of OSTBC for $\mathrm{Nr}=1$.

As we shown in the above figure represents the BER V/s SNR with constant $\mathrm{Nr}=1$. In this case G8 shows the best performance compared to the G2,G3,G4, H3 and H4. Compare to theG8 of QPSK G2, G3, H3, G4, H4 of QPSK is high SNR, theG8 of 16-QAM and 64-QAM G2, G3, H3, G4, H4 of 16-QAM and 64-QAM are high SNR also, so that the results shown that G8 is best performance compare to the other. G8 having the low SNR value so that it is the best performer when compare to the other.

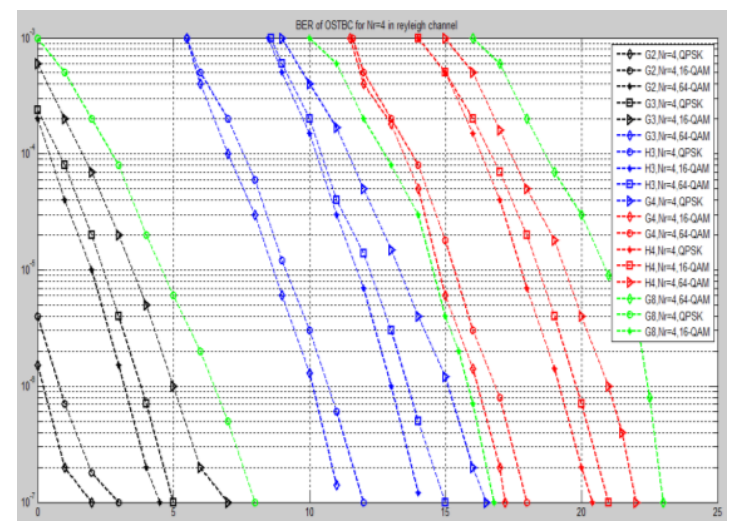

Fig.8 Bit error rate versus SNR of OSTBC for $\mathrm{Nr}=4$.

As we shown in the above figure represents the BER V/s SNR with constant $\mathrm{Nr}=4$. In this case G8 shows the best performance compared to the G2, G3, G4, H3 and H4. Compare to theG8 of QPSK G2, G3, H3, G4, H4 of QPSK is low SNR, theG8 of 16-QAM and 64-QAM G2, G3, H3, G4, H4 of 16-QAM and 64-QAM are low SNR also, so that the results shown that G8 is best performance compare to the other. G8 having the high SNR value so that it is the best performer when compare to the other. 


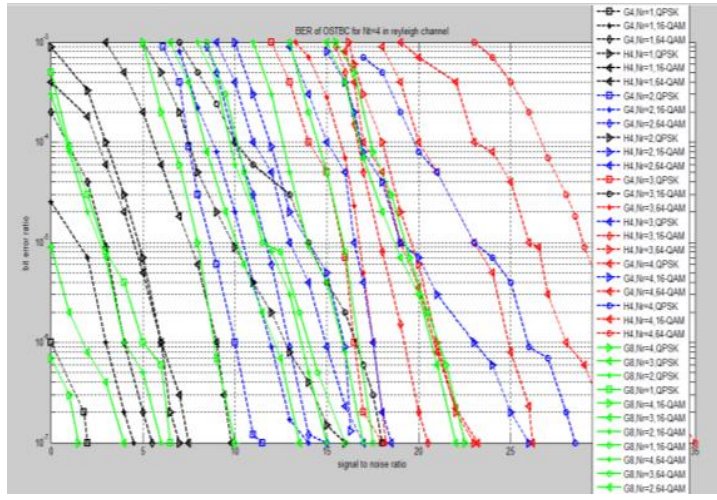

Fig.9 Bit error rate versus SNR of OSTBC for $\mathrm{Nt}=4$

As we shown in the above figure represents the BER V/s SNR with different $\mathrm{Nr}=1,2,3,4$. In this case G8 shows the best performance compared to the G2,G3,G4, H3 and H4. Compare to theG8 of QPSK G2, G3, H3, G4, H4 of QPSK is high SNR, theG8 of 16-QAM and 64-QAM G2, G3, H3, G4, H4 of 16-QAM and 64-QAM are high SNR also, so that the results shown that G8 is best performance compare to the other. G8 having the low SNR value so that it is the best performer when compare to the other.

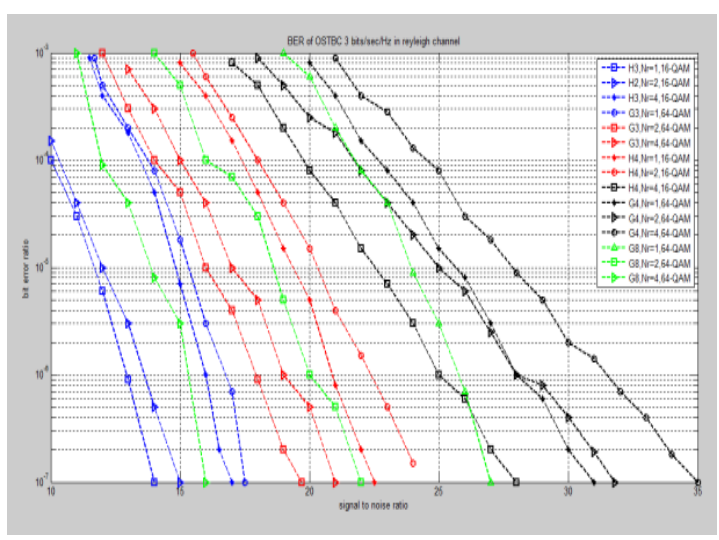

Fig.10 Bit error rate versus SNR of OSTBC at 3 bits/sec/Hz.

As we shown in the above figure represents the BER V/s SNR with different $\mathrm{Nr}=1,2,4$. In this case G8 shows the best performance compared to theG3,G4. Compare to theG8, 64QAM G3, G4 of 64-QAM are low SNR, so that the results shown that G8 is best performance compare to the other. G8 having the high SNR value so that it is the best performer when compare to the other.

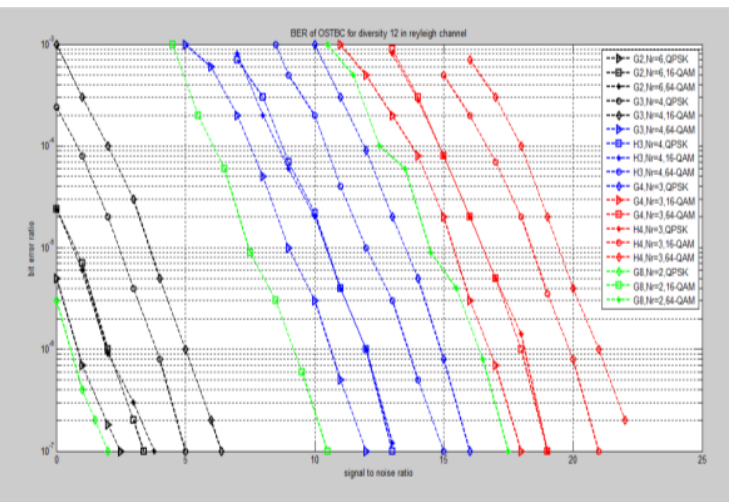

Fig.11 Bit error rate versus SNR of OSTBC with spatial diversity 12.
As we shown in the above figure represents the BER V/s SNR with different $\mathrm{Nr}=6,4,3,2$. In this case G8 shows the best performance compared to the G2, G3, G4, H3 and H4. Evaluate to theG8 of QPSK G2, G3, H3, G4, H4 of QPSK is high SNR, theG8 of 16-QAM and 64-QAM G2, G3, H3, G4, H4 of sixteen-QAM and sixty four-QAM are excessive SNR also, so that the results shown that G8 is first-rate efficiency compare to the other. G8 having the low SNR value so that it is the exceptional performer when compare to the opposite. Expand the number of $\mathrm{Nt}$ transmit antennas on the base station than increasing the quantity of $\mathrm{Nr}$ obtain antennas in any respect mobile stations.

\section{CONCLUSION}

This was especially proven when G3 outperformed all others for equal range obtain. The penalty of having more transmit antennas, which consequently reduces the power per transmit antenna was once observed. Also, we determined diminishing returns for every scheme because the number of bought antennas elevated. It was once specifically interesting to search out that even though $\mathrm{H} 3$ and $\mathrm{H} 4$ have better cost than G3 and G4, the efficiency of G3 and G4 is bigger and might consequently be favored in some situations. In the end, we conclude that it's most desirable to make use of a low constellation order with high code fee than excessive constellation order with low code expense.

\section{REFERENCES}

[1] A. Molisch, Wireless Communications. Wiley-IEEE Press, 2005.

[2] J. Winters, "On the capacity of radio communication systems with diversity in a Rayleigh fading environment," IEEE Journal on Selected Areas in Communications, vol. 5, no. 5, pp. 871-878, 1987.

[3] D. Gesbert, M. Shafi, D. Shiu, P. Smith, and A. Naguib, "From theory to practice: an overview of MIMO spacetime coded wireless systems," IEEE Journal on selected areas in Communications, vol. 21, no. 3, pp. 281-302, 2003.

[4] G. Tsoulos, MIMO system technology for wireless communications. CRC Press, 2006

[5] L. Dai, S. Zhou, H. Zhuang, and Y. Yao, "Closed-loop MIMO architecture based on water-filling," Electronics Letters, vol. 38, no. 25, pp. 1718-1720, 2002.

[6] K. Zheng, L. Huang, W. Wang, and G. Yang, "TDCDM-OFDM: Evolution of TD-SCDMA toward 4G," IEEE Communications Magazine, vol. 43, no. 1, pp. 45$52,2005$.

[7] H. Sampath, S. Talwar, J. Tellado, V. Erceg, and A. Paulraj, "A fourth-generation MIMO-OFDM broadband wireless system: design, performance, and field trial results," IEEE Communications Magazine, vol. 40, no. 9, pp. 143-149, 2002.

[8] “IEEE P802.11n/D5.0,” May 2008.

[9] H. Niu and Ngo, "Diversity and Multiplexing Switching in 802.11 n MIMO Systems," in Signals, Systems and Computers, 2006.ACSSC'06. Fortieth Asilomar Conference on, 2006, pp. 1644-1648.

[10] G. Foschini and M. Gans, "On limits of wireless communications in a fading environment when using multiple antennas," Wireless personal communications, vol. 6, no. 3, pp. 311-335, 1998. 
[11] V. Tarokh, H. Jafarkhani, and A. Calderbank, "Spacetime block coding for wireless communications: performance results," IEEE Journal on selected areas in communications, vol. 17, no. 3, pp. 451-460, 1999.

[12] T. Kaiser, Smart Antennas-State of the Art. Hindawi Publishing Corporation, 2005.

[13] A. Molisch, "A generic model for MIMO wireless propagation channels in macro-and microcells," IEEE Transactions on Signal Processing, vol. 52, no. 1, pp. 6171,2004
[14] S. Alamouti, "A simple transmit diversity technique for wireless communications," IEEE Journal on selected areas in communications, vol. 16, no. 8, pp. 1451-1458, 1998.

[15] V. Tarokh, H. Jafarkhani, and A. Calderbank, "Spacetime block codes from orthogonal designs," IEEE Transactions on Information Theory, vol. 45, no. 5, pp. 1456-1467, 1999.

[16] L. Cortes-Pena, "Orthogonal Space-Time Coding Matlab Source Files," Software online: http:// users.ece.gatech.edu/_Cortes/STBC Matlab.html, 2009. 